\title{
ANALYSIS OF THE SUN'S OBSERVATIONS WITH PRISMATIC ASTROLABE
}

\author{
N.V.LEISTER, P.C.R.POPPE, M.EMILIO AND F.LACLARE \\ Inst.Astron.Geof.USP, Caixa Postal 9638, 01065, Brazil \\ Observatoire de la Côte D'Azur, 06460 Saint Vallier, France
}

\section{Introduction}

A regular program of observations of the Sun with prismatic astrolabes is under way since 1974 in both, the "Abrahão de Moraes" $\left(\phi=-23^{\circ} 0.1^{\prime}\right)$ and "Caussols" $\left(\phi=+43^{\circ} 44.9^{\prime}\right)$ observatories. The zenith distance of 2968 transits of the solar limbs observed during the period from 1988 January to 1994 April, in both centers, are analysed. The primary goal of this programme is to determine corrections $\Delta \mathrm{E}$ and $\Delta \mathrm{A}$ to the FK5 equinox and equator, as well as to the Earth's orbital constants. Secondly, we present here possible variations of the solar radius, which are of interest because of their astrophysical significance. The results of the analysis of the observations made with these instruments depend on both the prism angles used during the observation and the latitude of the site (Leister 1989, Poppe 1994). With the use of the different reflector prisms, instead of the refracting equilateral, it was possible to observe at differents zenith distances, allowing to sweep, at the brazilian observatory, the apparent orbit of the Sun during a period of about 10 months per year, with two prisms. The instrument of the french observatory works with 11 differents zenith distances, permitting an increase of about 10 months per year in the observational period either.

\section{Orientation of the frame system}

The method of the data reduction is based upon the comparasion between the observed zenith distance obtained by means of the transit time and the zenith distance defined by the prisms. The analysis here developed in the same way that adopted by Leister (Leister 1989) and could be made directly by mean of the condition equation: 


$$
\Delta \mathrm{z}=\mathrm{f}(\Delta \mathrm{E}, \Delta \mathrm{A})+\mathrm{g}(\Delta \mathrm{L}, \Delta \epsilon, \Delta \mathrm{h}, \Delta \mathrm{k}),
$$

where $\mathrm{f}=\cos \phi \sin \mathrm{Z} \Delta \mathrm{E}+\cos \mathrm{Z} \Delta \mathrm{A}$ and

$$
\begin{aligned}
\mathrm{g}= & (\cos S \sin \epsilon \cos \alpha+\sin S \cos \epsilon \sec \delta) \Delta \mathrm{L} \\
& +(\cos S \sin \alpha-\sin S \cos \alpha \sin \delta) \Delta \epsilon \\
& +2\left(\sin \delta \cos S \cos ^{2} \alpha+\sin S \sin \alpha\right) \Delta \mathrm{h} \\
& -2(\cos S \sin \epsilon \cos \delta \cos \alpha+\sin S \cos \epsilon \cos \alpha) \Delta \mathrm{k} .
\end{aligned}
$$

$\mathrm{S}$ is the paralactic angle and $\mathrm{Z}$ is the azimuth.

The solution, together with the standard errors, calculated in the usual way from the variance-covariance matrix, is displayed in table 1 .

The variance of the solution is 0.60 ", giving a standard error of 0.78 " for an observation of unit weight. The value of latitude and clock correction, that we utilized here, was provided by the International Earth Rotation Service (IERS). The mean values of the latitude and longitude were refered to the FK5 reference system, obtained for the stars observations.

TABLE 1. General solution

\begin{tabular}{ll} 
& (arcseconds) \\
\hline$\Delta \mathrm{E}$ & $+0.10 \pm 0.04$ \\
$\Delta \mathrm{A}$ & $+0.07 \pm 0.07$ \\
$\Delta \mathrm{L}$ & $-0.37 \pm 0.03$ \\
$\Delta \epsilon$ & $+0.63 \pm 0.07$ \\
$\Delta \mathrm{e}$ & $+0.04 \pm 0.01$ \\
$\mathrm{e} \Delta \pi$ & $-0.37 \pm 0.08$ \\
\hline Dispersion & 0.78 \\
$\mathrm{~N}$ & 1473 \\
Mean date & 1990.7 \\
\hline
\end{tabular}

\section{Solar diameter measurements}

The solar diameter follows directly from the timing of the limb transits. Here, we present the results of the diameter measurements from 1980 September to 1993 , comprising 5,000 limbs observations. The advantage of prismatic astrolabes is that the observations are not affected by error in atmospheric refraction $r^{\prime}$. An error in $r^{\prime}$ affecting the apparent zenith distance cancels out, as the radius is half the difference between the zenith distances of upper and inner solar edges. The correction $\mathrm{R}$ for the apparent diameter can be written:

$$
\mathrm{R}=15 \cos \phi \sin \mathrm{Z}\left(\Delta t_{o}-\Delta t_{c}\right)+\mathrm{C}+\mathrm{kF}
$$

where $\mathrm{C}$ denote the sum of measured corrections arising from small refraction variations and geometrical effects; $\mathrm{F}$ is unknown plate prismatic error 


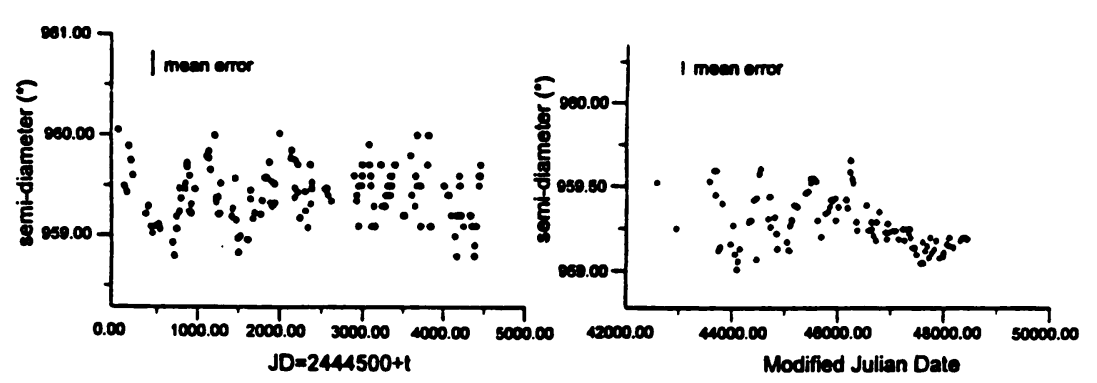

Figure 1. Monthly means of the apparent solar radius obtained at "Abrahão de Moraes" and "CERGA" observatories

and the value of the $\mathrm{k}(+1$ or -1$)$ depend on the initial plate position. The above equation was solved for the unknows $\mathrm{R}$ and $\mathrm{F}$ at monthly intervals, with an average of 20 transits for the brazilian set and 60 transits for the french set. The results are displayed in Figures 1 and 2 for the same intervals.

TABLE 2.

The average observed solar semi-diameter

\begin{tabular}{ll}
\hline set & $R(”)$ \\
\hline brazilian set & $959.40 \pm 0.05$ \\
french set & $959.37 \pm 0.02$ \\
\hline
\end{tabular}

The standard deviation of the monthly means is about 0.30 " for the brazilian program. The CERGA program allows a large number of observations each day so the standard deviation of daily average is 0.15 ".

\section{Conclusion and remarks}

Concerning the determination of the equator and equinox corrections the solution obtained by least square method reveals the correction $0.10 " \pm 0.04$ " and $0.07 " \pm 0.07 "$ to equinox and equator respectively, at mean epoch of observations 1990.7 . The good quality of this data set shows that the FK5 do not support a significant correction. For all series of data analysed, we obtained important corrections of the obliquity of the ecliptic. In spite of the different values of the dispersion to be found among the set data of the measurements of the semi-diameters, a sinusoidal component is visible on the plots of Figure 1. A harmonic analysis reveals a significant term of period 1,000 \pm 50 days (Leister et al 1990, Laclare 1983). The amplitude of 


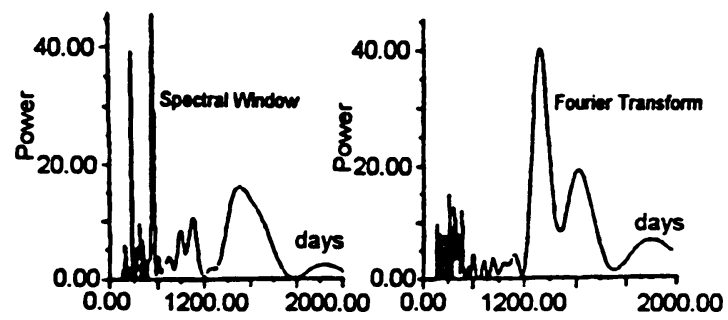

Figure 2. Fourier Transform and Spectral Window - brazilian set

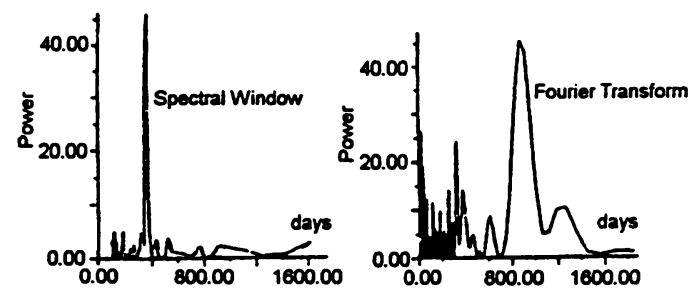

Figure 3. Fourier Transform and Spectral Window - french set

the signal in both cases is 0.25 ", so the signal is similar, in amplitude and phase (Figure 2 and 3 ). The main difference between the two time series does not lie in the thecnique but rather in the geographic location of the observatories. A second difference could be the heliographic latitudes of the apparent radius measured at the two sities; this fact could suggest a phenomenon of radial pulsation.

\section{Acknowledgements}

This research was supported by FAPESP (grants $n^{0} \quad 92 / 3762-0$ and $n$ ? 95/0723-2)

\section{References}

Leister, N.V. (1989) Orientação do sistema de referência. Observações do Sol com o astrolábio de Valinhos, Ph.D.Thesis, IAGUSP.

Poppe, P.C.R. (1994) Análise das observações do Sol com o astrolábio solar no período de 1988-1992, Msc. Thesis, IAGUSP.

Laclare, F. (1983) Measures du diamètre solaire à l'astrolabe, $A \& A$ 125, pp. 200-203

Leister, N.V. and Benevides-Soares, P. (1990) Variations du diamétre solaire, C.R.Acad.Sci. Paris 311, pp. 399-404 